\title{
Aplicação da matriz FPSEEA de saúde ambiental para a macrometrópole paulista: subsídios para o planejamento e gestão socioambiental regional
}

\author{
Application of the DPSEEA environmental health matrix for paulista macrometrópole: subsidies \\ for regional socio-environmental management and planning
}

\author{
Natasha Ceretti Maria ${ }^{1}$ \\ Anne Dorothée Slovic ${ }^{2}$
}

\begin{abstract}
Resumo
Ao planejamento da gestão urbana em escalas regionais faz-se necessário incorporar a dimensão ambiental para que as cidades possam enfrentar os desafios decorrentes da reestruturação do espaço pelas dinâmicas econômicas e a adaptação aos efeitos das mudanças climáticas. Nesse contexto, a Macrometrópole Paulista (MMP) apresenta heterogênea divisão funcional socioambiental entre os seus municípios. Essas divisões corroboram com diferentes condições socioambientais nesse conjunto regional que podem ser expressas por indicadores estratégicos que qualifiquem as localidades municipais. Para tal, este trabalho tem como objetivo selecionar indicadores relevantes no contexto da saúde ambiental e da sustentabilidade para os municípios que compõem a Macrometrópole Paulista. Para a seleção dos indicadores foi considerada a matriz de indicadores para a Saúde Ambiental denominada de FPSEEA (Força Motriz-Pressão-Situação-Exposição-EfeitoAções). Os resultados podem ser vistos como uma nova maneira inovadora de qualificar as condições socioeconômicas, ambientais e de saúde, destacando a importância da escala regional como uma nova unidade territorial a ser explorada na gestão urbana.
\end{abstract}

Palavras-chave: Macrometrópole. Saúde ambiental. Sustentabilidade. Indicadores. São Paulo.

\begin{abstract}
The planning of urban management at regional scales makes it necessary to incorporate the environmental dimension so that cities can face the challenges arising from the restructuring of space by economic dynamics and adaptation to the effects of climate change. In this context the Macrometropole Paulista (MMP) presents a heterogeneous social and environmental functional division between its municipalities. These divisions corroborate with different socio-environmental conditions in this regional set that can be expressed by strategic indicators that qualify the municipalities. To this end, this study aims to select relevant indicators in the context of environmental health and sustainability for the municipalities that make up the MMP. For the selection of the indicators, the matrix of indicators for Environmental Health was defined as DPSEEA (Driving Force-Pressure-Situation-Exposure-Effect-Actions). The results can be seen as a new innovative way of qualifying socioeconomic, environmental and health conditions, highlighting the importance of the regional scale as a new territorial unit to be explored in urban management.
\end{abstract}

Keywords: Macrometropole. Environmental health. Sustainability. Indicators. São Paulo.

\footnotetext{
${ }^{1}$ Faculdade de Saúde Pública (FSP-USP). E-mail: natashaceretti@ yahoo.com.br

${ }^{2}$ Instituto de Geofísicas e Astronomia (IAG-USP). E-mail: adslovic@usp.br
} 


\section{Introdução}

Os sistemas regionais podem ser considerados como sistemas adaptativos complexos e heterogêneos. Assim, o desenvolvimento do conhecimento sobre sistemas metropolitanos em uma escala regional pode, teoricamente, promover respostas adequadas e contribuir para a capacidade de planejar megacidades mais sustentáveis (STEINER, 2004; WU, 2014).

Frente ao atual cenário fortemente marcado por mudanças climáticas e as incertezas decorrentes destas, bem como tendências de alterações nas dinâmicas de regime hídrico, torna-se urgente a necessidade de avançar no conhecimento e na inovação associada à adoção de medidas flexíveis de gestão adaptativa e implementação de práticas de planejamento e gestão socioambiental numa perspectiva integrada e interdependente.

Face às evidências do aquecimento global e as consequências na variabilidade climática, além da atual crise hídrica metropolitana, surge a proposta de selecionar indicadores relevantes em um contexto de Saúde Ambiental e de Sustentabilidade para uma escala regional que transcende a Região Metropolitana de São Paulo (RMSP), cuja denominação mais difundida é o termo Macrometrópole Paulista (MMP). Segundo Asquino (2010), uma das características do planejamento de sistemas de infraestrutura regionais em São Paulo é o reconhecimento da MMP como recorte necessário para análise de benefícios e de impactos de políticas públicas.

O contexto atual para a gestão urbana sustentável exige considerar a dimensão ambiental que faz as megacidades enfrentarem novos desafios devido à reestruturação do espaço com as novas dinâmicas econômicas e dos possíveis riscos das alterações climáticas (CHAE, 2010). Nesse sentido, o primeiro passo é produzir diagnósticos que podem orientar o planejamento de políticas públicas, considerando a heterogeneidade das municipalidades de sistemas regionais.

A seleção da escala macrometropolitana como objeto de estudo não é uma simples abordagem de expansão da metrópole, mas se trata do reconhecimento de um novo patamar de interação territorial funcional e de suas interdependências, que demanda ampla revisão dos paradigmas existentes em torno dos instrumentos setoriais e locais (SILVA et al., 2012).

Assim, este estudo propõe identificar e selecionar indicadores provenientes de dados disponibilizados por instituições públicas adequados ao contexto da MMP, que possam preencher as dimensões de uma matriz analítica em Saúde Ambiental.

Há poucos estudos metropolitanos dirigidos a megacidades que foquem a fragmentação do território, sua heterogeneidade e interdependências, de forma necessariamente equilibrada às dimensões de sustentabilidade de grandes conglomerados urbanos (MARIA, 2015). Partindo dessa 
premissa, esperando contribuir com o estudo de aspectos relacionados com a gestão e o planejamento socioambiental em uma escala regional, a pesquisa pode aprimorar a aplicação de uma tipologia como um instrumento metodológico capaz de auxiliar a gestão e formulação de políticas públicas para lidar com os desafios e as exigências do contexto macrometropolitano paulista.

A análise das condições e tendências das interações entre forças motrizes (entendidas como os fatores que em escala macro influenciam os processos ambientais que podem afetar a saúde humana) e pressões socioeconômicas por meio da estruturação de sistemas de indicadores constitui um desafio para a Saúde Pública (FREITAS; GIATTI, 2009), uma vez que os problemas ambientais são, simultaneamente, problemas de saúde, afetando os seres humanos e as sociedades em múltiplas escalas e dimensões.

Após as Conferências de Estocolmo e a Eco-92, influenciadas pelas questões levantadas pelo movimento ambientalista, emergiu em paralelo uma Saúde Pública que tem como estratégia mudar o foco das práticas centradas nos aspectos biomédicos da atenção para uma compreensão preventiva do estado de saúde. Essa tendência atualmente é fortemente corroborada com a proposição dos Objetivos de Desenvolvimento Sustentável da Organização das Nações Unidas (FREITAS, 2003; KICKBUSH; BUSS, 2014; PNUD, 2015).

Nesse contexto, indicadores de saúde ambiental e de sustentabilidade organizados em uma matriz dimensional podem ser utilizados como ferramenta metodológica para qualificar as localidades municipais, buscando identificar similaridades e assimetrias socioambientais entre as cidades. Desconsiderar as similaridades, as diferenças e a heterogeneidade de cada localidade dos sistemas metropolitanos pode determinar o fracasso e a insustentabilidade das políticas públicas quando estas consideram as cidades de maneira isolada.

Assim, justifica-se um estudo utilizando uma matriz de indicadores de Saúde Ambiental como uma ferramenta de auxílio para a compreensão das relações de um sistema macrometropolitano, destacando a importância da escala regional como uma nova unidade territorial a ser explorada na busca de instrumentos e soluções que sejam mais adequados para lidar com os desafios e as exigências dos problemas contemporâneos.

Este estudo tem como objetivo identificar e selecionar um conjunto de indicadores com relevância nos contextos de saúde ambiental e de desenvolvimento sustentável (DS) para a MMP, conforme proposto pelo modelo de organização ou sistema de indicadores Força Motriz-PressãoSituação-Exposição-Efeito-Ações (FPSEEA) elaborado pela organização Mundial de Saúde em conjunto com o Programa das Nações Unidas para o Meio Ambiente (PNUMA). 


\section{Metodologia}

\section{Dados Gerais da área de estudo}

A Macrometrópole Paulista (MMP) (Fig. 1) é um dos maiores aglomerados urbanos do Hemisfério Sul, abrigando a Região Metropolitana de São Paulo que está entre as seis maiores do mundo (EMPLASA, 2012). É a região de maior importância socioeconômica do país, pois esse extenso território concentra indústrias de alta tecnologia, comércio diversificado, os serviços mais complexos e a agroindústria mais produtiva. Os maiores portos e aeroportos, o melhor complexo rodoviário e os maiores polos de conhecimento e inovação. Estão inseridas nessa área cinco regiões metropolitanas: São Paulo (RMSP), Baixada Santista (RMBS), Campinas (RMC), Vale do Paraíba e Litoral Norte (RMVPLN), Sorocaba (RMS) e Ribeirão Preto (RMRP). Duas aglomerações urbanas: Jundiaí (AUJ) e Piracicaba (AUP), além da Unidade Regional Bragantina (URB) (Bragantina-São Roque), ainda não institucionalizada (Fig. 2) (EMPLASA, 2012).

Esta ainda considera as áreas de oito Unidades de Gerenciamento de Recursos Hídricos (UGRHIs) que compõem a organização estadual para a gestão de recursos hídricos (SSRH/DAEE/COBRAPE, 2013).

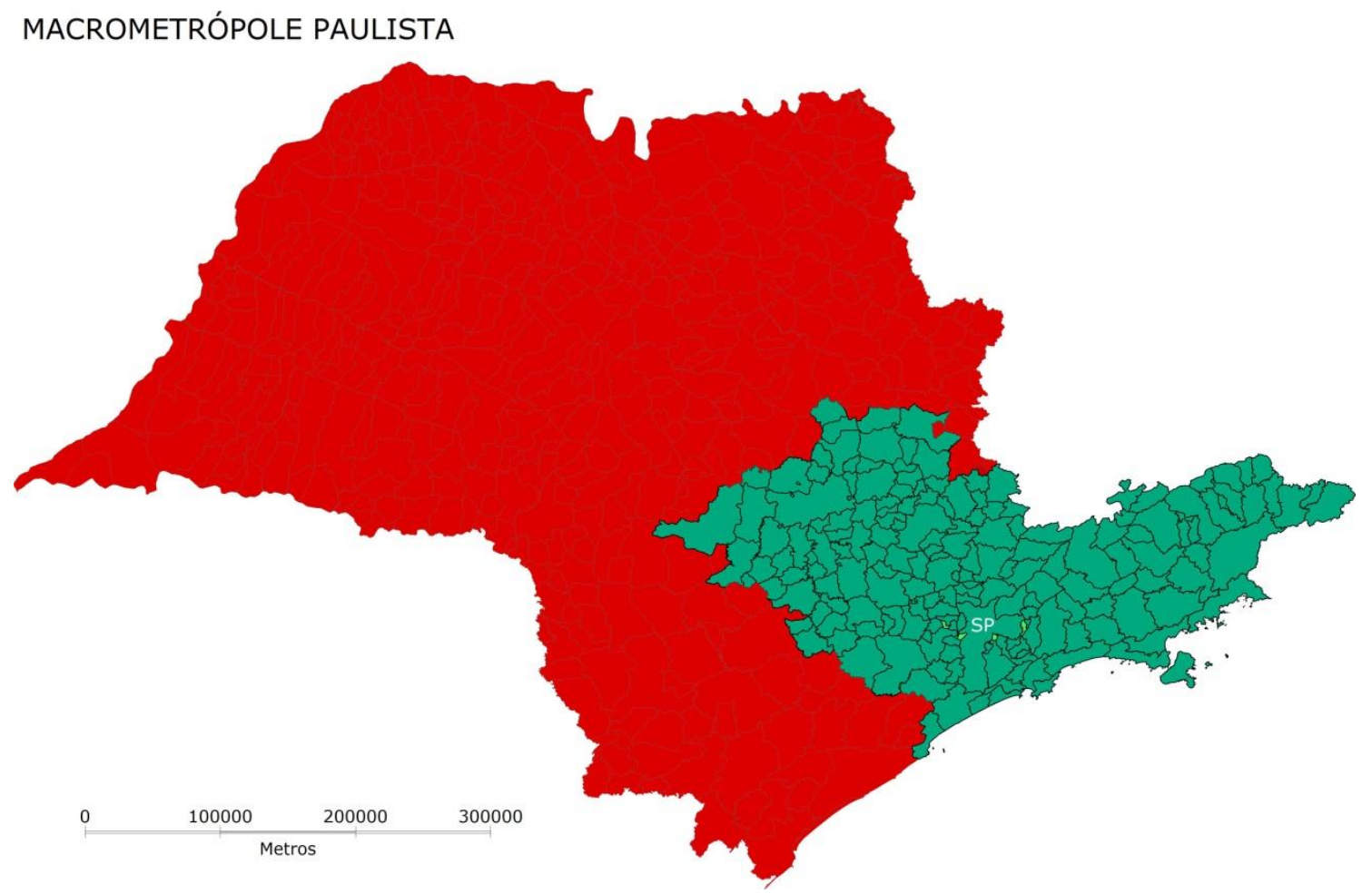

FIGURA 1 - MACROMETRÓPOLE PAULISTA

FONTE: Elaboração própria. 


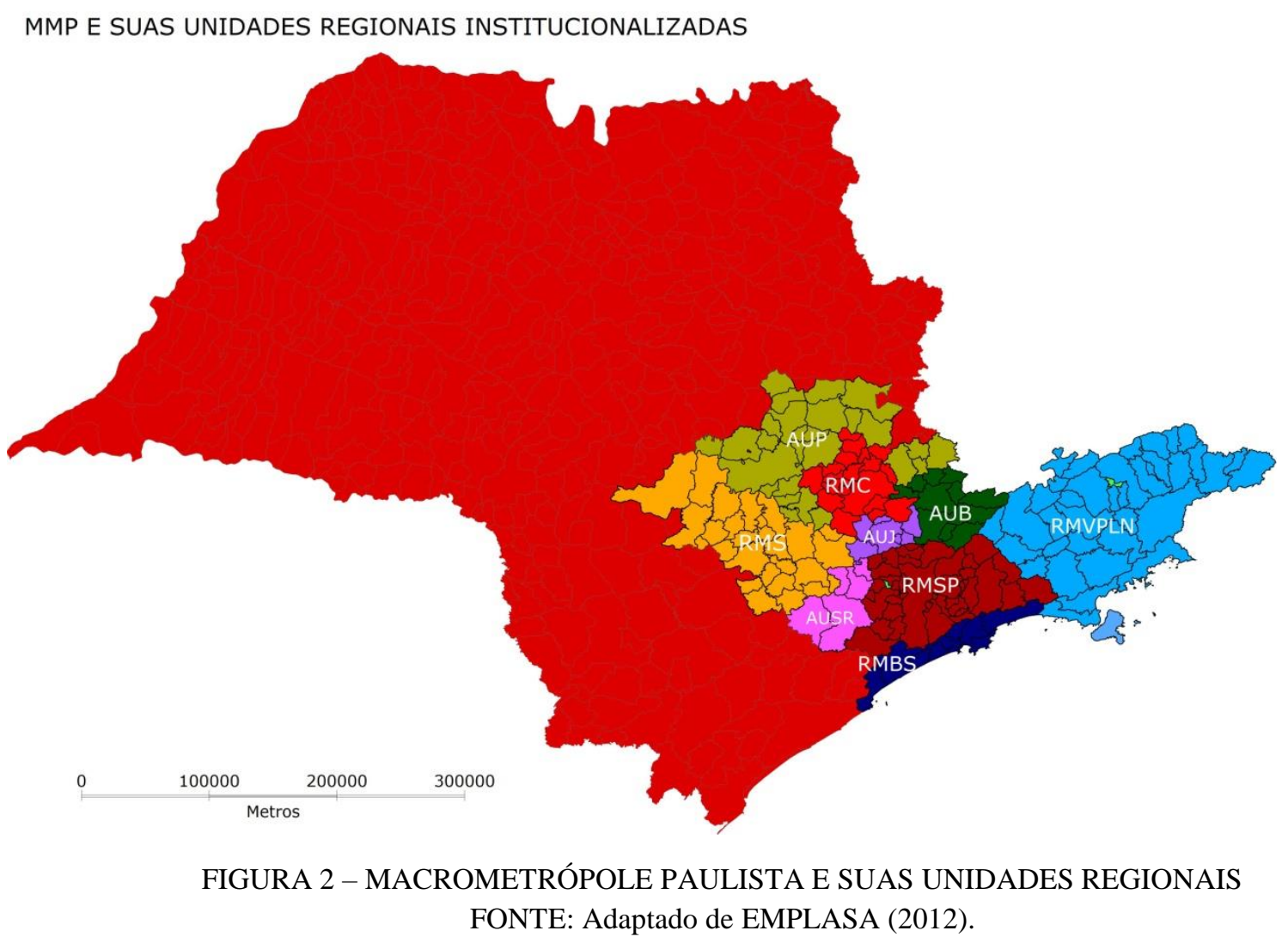

\section{Seleção dos indicadores}

O estudo foi realizado por meio de dados secundários obtidos a partir de pesquisa documental sobre indicadores fornecidos por instituições públicas e revisão bibliográfica sobre o tema de estudo.

As principais fontes utilizadas para a seleção dos indicadores foram as bases de dados governamentais de acesso aberto: Instituto Brasileiro de Geografia e Estatística (IBGE), Departamento de Informática do SUS/Ministério da Saúde (DATASUS/MS), Fundação Sistema Estadual de Análise de Dados (SEADE), Programa das Nações Unidas Para o Desenvolvimento (PNUD), Instituto Florestal (IF), Companhia Ambiental do Estado de São Paulo (CETESB), Secretaria de Energia e Mineração do estado de São Paulo (SE), Secretaria da Saúde do Estado de São Paulo (SES-SP), Centro de Vigilância Epidemiológica (CVE) e Datapedia.

Lançada no ano de 2017, o Datapedia é uma janela única de dados públicos e oficiais de todos os municípios e distritos do país que foi organizada pelo Núcleo de Geotecnologias (Sistema Labgis) da Universidade do Estado do Rio de Janeiro (UERJ). São 5.570 localidades presentes na 
plataforma, que agrupou, organizou e traduziu em visualização didática as mais diversas fontes de dados com o objetivo de aumentar a transparência da gestão pública.

Para a seleção de alguns dos indicadores da tipologia foi considerada a matriz FPSEEA de indicadores para a saúde ambiental desenvolvida em conjunto com o Programa das Nações Unidas para o Meio Ambiente (PNUMA), pela Organização Mundial de Saúde (OMS) e pela Agência de Proteção Ambiental dos Estados Unidos (USEPA). A matriz estabelece um fluxo de demandas e pressões por recursos naturais e alterações nos ecossistemas, possibilitando um amplo olhar para as cadeias de causas e consequências que interferem na saúde e bem-estar humanos. Por possuir múltiplas dimensões, permite analisar desde as forças motrizes $(\mathrm{F})$ às pressões $(\mathrm{P})$ que interferem no estado/situação do ambiente (S), modulando as exposições (E) dos humanos a doenças, que constam como os efeitos finais (E) na cadeia. Portanto, sob essa visão sistêmica e hierarquizada sobre os problemas de saúde e do ambiente, distintas formas de ação (A) são passíveis de execução em diferentes níveis, assim como também são possíveis variadas formas de controle e prevenção (CORVALÁN e col., 2000). Segundo o Manual de Indicadores do Ministério da Saúde, de Sobral e col. (2011), o modelo proposto pela OMS parece apropriado para o estabelecimento de uma base racional capaz de expor, de forma estruturada, uma matriz de indicadores integrantes da cadeia relacional entre os determinantes socioambientais e eventos de saúde (Figura 3).

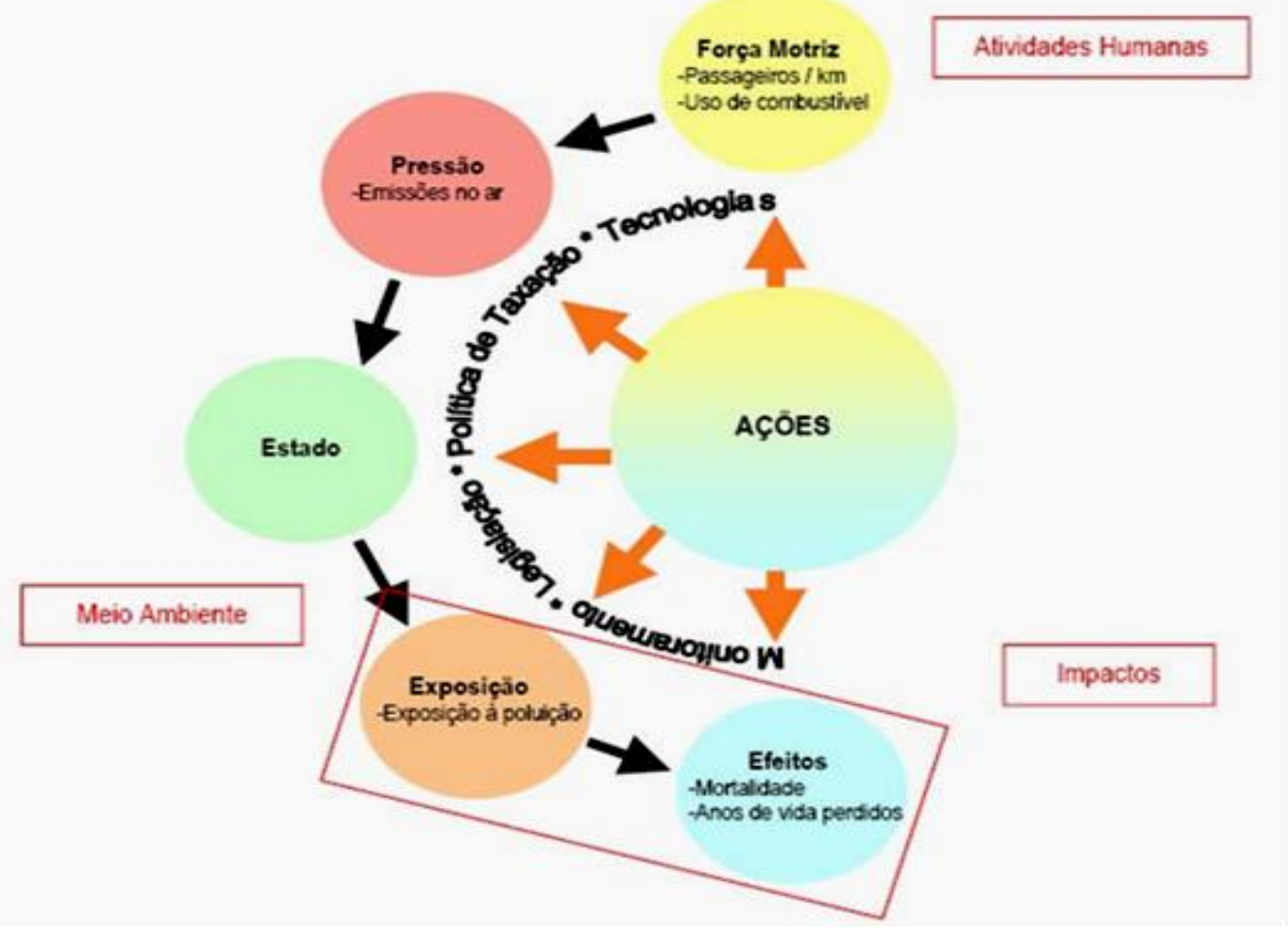

FIGURA 3 - MATRIZ FPSEEA

FONTE: Adaptado de CORVALÁN e col. (2000). 
A abordagem FPSEEA, adotada pela OMS, vem sendo aplicada no Brasil pela Coordenação Geral de Vigilância em Saúde Ambiental da Secretaria de Vigilância em Saúde do Ministério da Saúde (CGVAM/SVS/MS) desde o final da década de 1990. Os esforços para a construção de indicadores que apontem para as inter-relações de mudanças ambientais e situação de saúde se encontram na origem do processo de institucionalização da vigilância em saúde ambiental no âmbito do Setor Saúde na passagem do século 20 para o 21, inicialmente pelo Decreto $\mathrm{n}^{\circ} 3.450 / 2000$, da Presidência da República, estabelecendo no antigo Centro Nacional de Epidemiologia (Cenepi) a gestão do sistema nacional de vigilância epidemiológica e ambiental e, posteriormente, por meio da Instrução Normativa SVS nº 1, de 2005, que regulamentou o Subsistema Nacional de Vigilância em Saúde Ambiental (SINVSA) (SOBRAL et al., 2011). No Quadro 1 a seguir apresenta-se um conjunto de indicadores utilizado pela saúde ambiental, organizado segundo o modelo FPSEEA.

\section{TABELA 1 - EXEMPLOS DE INDICADORES DE SAÚDE AMBIENTAL ORGANIZADOS SEGUNDO O MODELO FPSEE}

\begin{tabular}{|c|c|c|c|c|c|}
\hline Força Motriz & Pressão & Situação & Exposição & Efeito & Ação \\
\hline $\begin{array}{l}\text { Índice de Gini da } \\
\text { distribuição do } \\
\text { rendimento } \\
\text { mensal de pessoas } \\
\text { de } 10 \text { anos ou } \\
\text { mais de idade com } \\
\text { rendimento. }\end{array}$ & $\begin{array}{l}\text { Coleta de esgoto } \\
\text { sanitário } \\
\text { (percentual de } \\
\text { domicílios sem } \\
\text { serviço de rede } \\
\text { coletora de } \\
\text { esgotamento } \\
\text { sanitário e/ou } \\
\text { pluvial). }\end{array}$ & $\begin{array}{l}\text { Saneamento } \\
\text { inadequado } \\
\text { (percentual de } \\
\text { domicílios sem } \\
\text { condições } \\
\text { simultâneas de } \\
\text { abastecimento de } \\
\text { água por rede } \\
\text { geral, } \\
\text { esgotamento } \\
\text { sanitário por rede } \\
\text { geral e lixo } \\
\text { coletado } \\
\text { diariamente). }\end{array}$ & 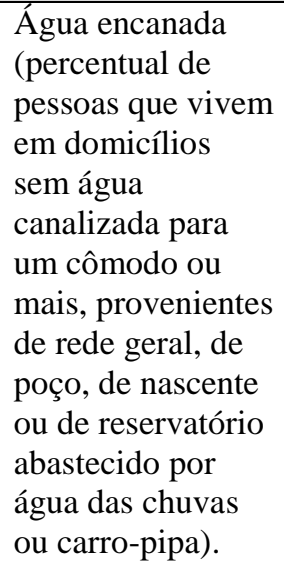 & $\begin{array}{l}\text { Taxa de } \\
\text { mortalidade } \\
\text { infantil (número } \\
\text { de óbitos de } \\
\text { menores de um } \\
\text { ano de idade, por } \\
\text { mil nascidos } \\
\text { vivos, na } \\
\text { população } \\
\text { residente em } \\
\text { determinado } \\
\text { espaço geográfico, } \\
\text { no } \\
\text { ano considerado). }\end{array}$ & $\begin{array}{l}\text { Existência de } \\
\text { conselhos } \\
\text { municipais de } \\
\text { Saúde e Meio } \\
\text { Ambiente. }\end{array}$ \\
\hline $\begin{array}{l}\text { Taxa de } \\
\text { crescimento } \\
\text { populacional } \\
\text { (diferença } \\
\text { percentual entre a } \\
\text { população em dois } \\
\text { ou mais anos } \\
\text { considerados). }\end{array}$ & $\begin{array}{l}\text { Tratamento de } \\
\text { esgoto } \\
\text { (percentual de } \\
\text { distritos sem } \\
\text { tratamento de } \\
\text { esgoto sanitário } \\
\text { coletado). }\end{array}$ & $\begin{array}{l}\text { Coleta de lixo } \\
\text { (percentual de } \\
\text { domicílios sem } \\
\text { serviço de coleta } \\
\text { direta ou indireta } \\
\text { regular de lixo, } \\
\text { inclusive } \\
\text { queimado ou } \\
\text { enterrado, jogado } \\
\text { em terreno baldio } \\
\text { ou logradouro, rio, } \\
\text { lago ou mar e } \\
\text { outros). }\end{array}$ & $\begin{array}{l}\text { Tratamento de } \\
\text { água } \\
\text { (volume em } \\
\text { percentual de água } \\
\text { distribuída por dia } \\
\text { sem tratamento). }\end{array}$ & $\begin{array}{l}\text { Internação } \\
\text { menores de } \\
\text { cinco anos por } \\
\text { DDA1 (taxa de } \\
\text { Internação } \\
\text { hospitalar por } \\
\text { DDA de crianças } \\
\text { menores de } 5 \text { anos } \\
\text { e a população } \\
\text { residente de } \\
\text { menores de } 5 \\
\text { anos, por } 1.000 \\
\text { crianças). }\end{array}$ & $\begin{array}{l}\text { Gastos públicos } \\
\text { com saúde, como } \\
\text { proporção do PIB } \\
\text { (percentual do } \\
\text { produto interno } \\
\text { bruto } \\
\text { que corresponde } \\
\text { ao gasto público } \\
\text { com saúde, } \\
\text { desagregado por } \\
\text { esfera de governo } \\
\text { - federal, estadual } \\
\text { e municipal). }\end{array}$ \\
\hline $\begin{array}{l}\text { Taxa de } \\
\text { urbanização } \\
\text { (percentual de } \\
\text { pessoas que vivem }\end{array}$ & $\begin{array}{l}\text { Consumo de } \\
\text { energia elétrica } \\
\text { (consumo }\end{array}$ & $\begin{array}{l}\text { Inundações ou } \\
\text { enchentes } \\
\text { (percentual de } \\
\text { municípios que }\end{array}$ & $\begin{array}{l}\text { Instalações } \\
\text { inadequadas } \\
\text { de esgoto } \\
\text { (percentual de } \\
\text { pessoas que vivem }\end{array}$ & $\begin{array}{l}\text { Internação } \\
\text { de menores de } \\
\text { cinco anos por } \\
\text { IRA2 (taxa de } \\
\text { internação }\end{array}$ & $\begin{array}{l}\text { Cobertura } \\
\text { populacional por } \\
\text { equipes de PSF3 e }\end{array}$ \\
\hline
\end{tabular}




\begin{tabular}{|c|c|c|c|c|c|}
\hline $\begin{array}{l}\text { em domicílios } \\
\text { urbanos). }\end{array}$ & $\begin{array}{l}\text { residencial per } \\
\text { capita de } \\
\text { eletricidade). }\end{array}$ & $\begin{array}{l}\text { sofreram } \\
\text { inundações ou } \\
\text { enchentes nos } \\
\text { últimos dois } \\
\text { anos). }\end{array}$ & $\begin{array}{l}\text { em domicílios } \\
\text { particulares } \\
\text { permanentes sem } \\
\text { acesso a } \\
\text { instalações } \\
\text { adequadas de } \\
\text { esgoto, ou seja, } \\
\text { que têm banheiro } \\
\text { de uso exclusivo e } \\
\text { com escoadouro } \\
\text { conectado à rede } \\
\text { coletora de esgoto } \\
\text { ou pluvial ou à } \\
\text { fossa séptica). }\end{array}$ & $\begin{array}{l}\text { hospitalar por } \\
\text { IRA de crianças } \\
\text { menores de } 5 \\
\text { anos e população } \\
\text { residente de } \\
\text { menores de } 5 \\
\text { anos, por } 1.000 \\
\text { crianças). }\end{array}$ & $\begin{array}{l}\text { ACS4 (percentual } \\
\text { da população } \\
\text { residente em } \\
\text { determinado } \\
\text { espaço } \\
\text { geográfico, no ano } \\
\text { considerado, que } \\
\text { recebe } \\
\text { atendimento } \\
\text { regular pelas } \\
\text { equipes de PSF e } \\
\text { ACS). }\end{array}$ \\
\hline $\begin{array}{l}\text { Renda familiar } \\
\text { per capita (total } \\
\text { de rendimentos de } \\
\text { uma família } \\
\text { dividido pelo } \\
\text { número de } \\
\text { pessoas da } \\
\text { família). }\end{array}$ & $\begin{array}{l}\text { Frota de } \\
\text { automóveis } \\
\text { por habitante } \\
\text { (razão entre a } \\
\text { frota total de } \\
\text { automóveis e o } \\
\text { número de } \\
\text { habitantes). }\end{array}$ & $\begin{array}{l}\text { Cobertura vegetal } \\
\text { (variação } \\
\text { percentual da } \\
\text { cobertura vegetal } \\
\text { em diferentes } \\
\text { estágios de } \\
\text { regeneração). }\end{array}$ & $\begin{array}{l}\text { Coleta de lixo } \\
\text { (percentual de } \\
\text { pessoas que vivem } \\
\text { em domicílios em } \\
\text { que a coleta de } \\
\text { lixo não é } \\
\text { realizada } \\
\text { diretamente por } \\
\text { empresa pública } \\
\text { ou privada, ou em } \\
\text { que o lixo não é } \\
\text { depositado em } \\
\text { caçamba, tanque } \\
\text { ou depósito fora } \\
\text { do domicílio). }\end{array}$ & $\begin{array}{l}\text { Taxa de } \\
\text { mortalidade por } \\
\text { causas externas } \\
\text { (número de óbitos } \\
\text { por causas } \\
\text { externas } \\
\text { - acidentes e } \\
\text { violência, por } 100 \\
\text { mil habitantes). }\end{array}$ & \\
\hline
\end{tabular}

FONTES: SOBRAL; FREITAS (2010); RIPSA (2008).

1 Doença diarreica aguda; 2 Infecção respiratória aguda; 3 Programa Saúde da Família; 4 Agentes Comunitários de Saúde.

Esse modelo permite a análise integrada da saúde ambiental dentro de um contexto econômico e social, podendo ser aplicado para subsidiar o monitoramento das condições de sustentabilidade ambiental e de saúde nos níveis regional e municipal (SOBRAL; FREITAS, 2010).

Além da matriz foram utilizadas como referências para critérios de seleção a publicação dos Indicadores de Desenvolvimento Sustentável do IBGE editado em 2017 e a Agenda 2030 para o Desenvolvimento Sustentável elaborada pela Organização das Nações Unidas (ONU) com os 17 Objetivos do Desenvolvimento Sustentável (ODSs) que contempla os seguintes temas: Erradicação da Pobreza; Fome Zero; Boa Saúde e Bem-Estar; Educação de Qualidade; Igualdade de Gênero; Água Limpa e Saneamento; Energia Acessível e Limpa; Emprego Digno e Crescimento Econômico; Indústria, Inovação e Infraestrutura; Redução das Desigualdades; Cidades e Comunidades Sustentáveis; Consumo e Produção Responsáveis; Combate às Alterações Climáticas; Vida de Baixo d'Água; Vida Sobre a Terra; Paz, Justiça e Instituições Fortes e Parcerias em Prol das Metas. 


\section{Resultados}

Com base na matriz são apresentados a seguir os indicadores selecionados em Saúde Ambiental para a MMP (Quadros 2, 3, 4, 5, 6 e 7). Apresenta-se uma lista detalhada dos indicadores correspondentes às múltiplas dimensões da matriz FPSEEA. Para cada indicador, apresenta-se sua definição correspondente contendo as informações que definem o indicador e a forma como ele se expressa, a fonte, o ano e as instituições responsáveis pela produção dos dados utilizados no seu cálculo e pelos sistemas de informação a que correspondem suas unidades de medida e, por fim, foi relacionado o tema de cada indicador selecionado com os 17 Objetivos do Desenvolvimento Sustentável (ODSs) e as dimensões do DS (Econômica, Social, Ambiental e Institucional).

O processo de seleção dos indicadores foi compatível com a adaptação da mesma aplicação da matriz de indicadores realizada pelo Ministério da Saúde para o Brasil e Unidades da Federação (SOBRAL et al., 2011), tendo como foco um recorte metropolitano (TALAMINI; GIATTI, 2013). As fichas de qualificação foram organizadas conforme metodologia estabelecida por (SOBRAL et al., 2011), considerando-se a conceituação do indicador, sua interpretação, usos, limitações, fontes e o método de cálculo. Por essas características citadas foram selecionados considerando sua relevância e abrangência em cada dimensão.

QUADRO 2 - INDICADORES SELECIONADOS QUE CORRESPONDEM À DIMENSÃO FORÇA MOTRIZ DA MATRIZ FPSEEA

\begin{tabular}{|lccccc}
\hline \multicolumn{1}{|c}{ Dimensão } & Indicador & Fonte & Unidade & Temática 0DSs & Dimensões DS \\
Força Motriz (FM1) & População & IBGE (Censo 2010) & Habitantes & ODS11 & Social \\
Força Motriz (FM2) & Tx. de Crescimento & Fundação SEADE & Porcentagem (\%) & ODS11 & Social \\
Força Motriz (FM3) & IDH-M & PNUD & & ODS3; ODS4; ODS8 & Social \\
Força Motriz (FM4) & Pop. Economicamente Ativa & IBGE (Censo 2010) & Porcentagem (\%) & ODS8 & Social \\
Força Motriz (FM5) & PIB- per capita & Fundação SEADE & Milhões & ODS8 & Econômica \\
Força Motriz (FM6) & Índice de Gini & DATASUS & & ODS1; ODS10 & Social \\
Força Motriz (FM7) & Domocilios com renda mensal per capita de até 1/2 salário mínimo & IBGE (Censo 2010) & Porcentagem (\%) & ODS10 & Social \\
Força Motriz (FM8) & Grau Urbanização & Fundação SEADE & Porcentagem (\%) & ODS11 & Social \\
Força Motriz (FM9) & Esperança de Vida ao Nascer & DATAPEDIA & Anos & ODS3 & Social \\
Força Motriz (FM10) & Média da Tx. de Fecundidade & DATAPEDIA & Porcentagem (\%) & ODS3; ODS11 & Social \\
Força Motriz (FM11) & Perc. de Mães Chefe de Família sem Fund. Completo com Filhos Menores de 15 anos & DATAPEDIA & Porcentagem (\%) & ODS4; ODS5; ODS10 & Social \\
\hline
\end{tabular}

FONTE: Elaboração própria. 
QUADRO 3 - INDICADORES SELECIONADOS QUE CORRESPONDEM À DIMENSÃO PRESSÃO DA MATRIZ FPSEEA

\begin{tabular}{|cccccc|}
\hline Dimensão & Indicador & Fonte & Unidade & Temática 0DSs & Dimensões DS \\
Pressão (P1) & Domicílios sem Ligação à Rede Pública de Esgoto (esgoto ou pluvial) & IBGE (Censo 2010) & Porcentagem (\%) & ODS6; ODS11 & Ambiental \\
Pressão (P2) & Frota de Veículos por Habitante & IBGE (Censo 2010) & Frota Total de Veículos & ODS11; ODS13 & Social \\
Pressão (P3) & Consumo de Energia Elétrica per capita & SE-SP & kWh & ODS7 & Econômica \\
Pressão (P4) & Produção de Lixo & CETESB & Tonelada/Dia & ODS12 & Ambiental \\
Pressão (P5) & Emissões de CO2 & SE-SP & Milhões de Toneladas & ODS13 & Ambiental \\
Pressão (P6) & Percental de População Vulnerável à Pobreza & DATAPEDIA & Porcentagem (\%) & ODS1; ODS10 & Social \\
Pressão (P7) & Percentual de População Abaixo da Linha de extrema Pobreza & DATAPEDIA & Porcentagem (\%) & ODS1; ODS10 & Social \\
Pressão (P8) & Percentual de população de 0 à 14 anos Vulnerável à Pobreza & DATAPEDIA & Porcentagem (\%) & ODS1; ODS10 & Social \\
\hline
\end{tabular}

FONTE: Elaboração própria.

\section{QUADRO 4 - INDICADORES SELECIONADOS QUE CORRESPONDEM À SITUAÇÃO DA MATRIZ FPSEEA}

\begin{tabular}{|lccccc|}
\hline \multicolumn{1}{|c}{ Dimensão } & Indicador & Fonte & Unidade & Temática ODSs & Dimensões DS \\
Situação (S1) & Domicílios Ligados à Rede Pública (esgoto ou pluvial) & IBGE (Censo 2010) & Porcentagem (\%) & ODS6; ODS11 & Ambiental \\
Situação (S2) & Domicílios com Coleta de Lixo & IBGE (Censo 2010) & Porcentagem (\%) & ODS11; ODS12 & Ambiental \\
Situaçãa (S3) & Índice de Qualidade de Aterro de Resíduos (IQR) & CETESB & Porcentagem (\%) & ODS11; ODS12 & Ambiental \\
Situação (S4) & Domicílios com Rede Geral de Distribuição de Água & IBGE (Censo 2010) & Porcentagem (\%) & ODS6; ODS11 & Ambiental \\
Situaçãa (S5) & Domicílios com Paredes Inadequadas & DATAPEDIA & Porcentagem (\%) & ODS11 & Social \\
Situação (S6) & Vegetação Natural Remanescente & IF & Porcentagem (\%) & ODS15 & Ambiental \\
Situação (S7) & Média do Percentual de População Atendida com Coleta Seletiva & SNIS (2010-2015) & Porcentagem (\%) & ODS11; ODS12 & Ambiental \\
\hline
\end{tabular}

FONTE: Elaboração própria.

QUADRO 5 - INDICADORES SELECIONADOS QUE CORRESPONDEM A SITUAÇÃO DA MATRIZ FPSEEA

\begin{tabular}{|cccccc|}
\hline \multicolumn{1}{|c}{ Dimensão } & Indicador & Fonte & Unidade & Temática 0DSs & Dimensões DS \\
Exposição (E1) & População sem Acesso à Rede de Abastecimento de Água & IBGE (Censo 2010) & Habitantes & ODS6; ODS11 & Ambiental \\
Exposição (E2) & População sem Instalação de Esgoto & IBGE (Censo 2010) & Habitantes & ODS6; ODS11 & Ambiental \\
Exposição (E3) & População com Abastecimento de Água e Esgotamento Sanitário Inadequado & DATAPEDIA & Habitantes & ODS6; ODS11 & Ambiental \\
Exposição (E4) & População sem Água Encanada e Banheiro & DATAPEDIA & Habitantes & ODS6; ODS11 & Ambiental \\
Exposição (E5) & Média do Percentual População sem Coleta de Lixo & DATAPEDIA & Habitantes & ODS11; ODS12 & Ambiental \\
\hline
\end{tabular}

FONTE: Elaboração própria.

QUADRO 6 - INDICADORES SELECIONADOS QUE CORRESPONDEM À SITUAÇÃO DA MATRIZ FPSEEA

\begin{tabular}{|cccccc|}
\hline Dimensão & Indicador & Fonte & Unidade & Temática ODSs & Dimensões DS \\
Efeito (EF1) & Média da Tx. de Mortalidade Geral & Fundação SEADE & Porcentagem (\%) & ODS3 & Social \\
Efeito (EF2) & Tx. de Mortalidade Infantil Abaixo de 1 ano & DATAPEDIA & Porcentagem (\%) & ODS3 & Social \\
Efeito (EF3) & Prevalência Média Anual de Dengue & SES & Porcentagem (\%) & ODS3 & Ambiental \\
\hline
\end{tabular}

FONTE: Elaboração própria. 
QUADRO 7 - INDICADORES SELECIONADOS QUE CORRESPONDEM À SITUAÇÃO DA MATRIZ FPSEEA

\begin{tabular}{|cccccc|}
\hline Dimensão & Indicador & Fonte & Unidade & Temática 0DSs & Dimensões DS \\
\hline Ações (A1) & Média do Percentual de Cobertura de Atenção Básica & DATAPEDIA & Porcentagem (\%) & ODS3; OSD17 & Institucional \\
Ações (A2) & Percentual de Gastos com Educação em Relação ao PIB Municipal & DATAPEDIA & Porcentagem (\%) & ODS4; ODS17 & Institucional \\
Ações (A3) & Percentual de Gastos com Urbanismo em Relação ao PIB Municipal & DATAPEDIA & Porcentagem (\%) & ODS11; ODS17 & Institucional \\
Ações (A4) & Gastos com Gestão Ambiental & DATAPEDIA & Sim/Não & ODS11; ODS17 & Institucional \\
Ações (A5) & 0 Municííio possui Política de Saneamento Básico Conforme a Lei 11445/2007 & SNIS & Sim/Não & ODS6; ODS17 & Institucional \\
Ações (A6) & O Município Adotou Parâmetros para Garantir à Saúde Pública & SNIS & Sim/Não & ODS3; ODS6; ODS17 & Institucional \\
Ações (A7) & 0 Município Possui Plano Municipal de Saneamento Conforme a Lei 11445/2007 & SNIS & Sim/Não & ODS6; ODS17 & Institucional \\
Ações (A8) & O Município é Integrante de Algum Consórcio Municipal & SNIS & Sim/Não & ODS6; ODS17 & Institucional \\
\hline
\end{tabular}

FONTE: Elaboração própria.

A escolha de cada indicador dentro de cada uma das dimensões propostas pela matriz baseou-se na tentativa de selecionar indicadores que representassem os parâmetros de influência das pressões antrópicas na qualidade ambiental urbana que interferem na saúde do ambiente.

Considerando-se os Quadros anteriores (2,3,4,5,6 e 7) com os indicadores selecionados para cada dimensão da matriz, por exemplo a Taxa de Mortalidade Infantil e a Prevalência Média Anual de Dengue, o procedimento de análise da matriz pode ser iniciado pelas "Forças Motrizes" com o tamanho da população e o elevado crescimento demográfico das áreas metropolitanas. Esse adensamento populacional vai gerar "Pressões" no meio ambiente como o aumento do consumo de recursos naturais, o aumento da produção de resíduos, aumento da emissão de $\mathrm{CO}^{2}$, déficit de saneamento pela falta de abastecimento de água e inexistência de coleta e tratamento de esgotos e lixo. Tais pressões vão gerar uma "Situação" ambiental de contaminação e deterioração dos recursos hídricos, da qualidade do ar, solo e perda de vegetação nativa. Este quadro facilita a "Exposição" humana a fatores de risco, podendo gerar "Efeitos" na saúde como o aumento da mortalidade infantil e o aumento de doenças por vetores tropicais como a dengue. Para cada uma das dimensões analisadas são propostas "Ações" de controle, promoção e, principalmente, prevenção da saúde, já que o modelo possui uma lógica precaucionária, em que as ações devem ser preferencialmente para intervir nas dimensões iniciais da matriz. Podem ser citadas como ações nesse sentido se o município possui uma Política de Saneamento Básico e se adota Parâmetros para garantir a Saúde Pública.

Como o modelo FPSEEA está apoiado no conceito de Desenvolvimento Sustentável e suas dimensões preconizadas pela Conferência das Nações Unidas sobre o Meio Ambiente e Desenvolvimento (Rio-92) (SACHS, 2004), os indicadores foram relacionados com os 17 Objetivos do Desenvolvimento Sustentável que integram a Agenda 2030 elaborada recentemente pela ONU para acompanhar o avanço das discussões mundiais nas questões que envolvem como se dará o 
desenvolvimento futuro. Sendo assim, fomentar ações que interfiram de forma positiva nas dimensões da matriz contribui para o alcance das metas almejadas.

Englobar todos esses aspectos faz com que o desenvolvimento seja tratado politicamente de forma integrativa, considerando toda a rede de causalidade entre ambiente, saúde e sustentabilidade.

\section{Discussão}

Ao abordar a MMP e a complexidade de seus problemas em uma escala regional por meio de um sistema de indicadores consagrados, grandes desafios emergem a partir desse debate. Quais indicadores são representativos da qualidade do meio ambiente em áreas urbanas e se relacionam com a saúde ambiental e a sustentabilidade do conjunto macrometropolitano? Essa resposta não é consensual e dependendo da opção metodológica adotada leva a resultados distintos.

A complexidade das realidades locais, distritais, municipais e metropolitanas, espaços que se ajustam e conflitam problemas socioambientais tão distintos (SILVA; SOUZA-LIMA, 2010), como é o caso da MMP, coloca a urbanização em xeque e conduz a repensar o desenvolvimento urbano de forma sustentável.

A indagação sobre o desenvolvimento sustentável e a sustentabilidade leva a formulações estratégicas para o alcance de melhores condições nesse processo que resultam em políticas públicas. Estas, por sua vez, devem ter parâmetros de mensuração para que o acompanhamento, no decorrer do tempo, seja realizado, e isso ocorre pela utilização de indicadores. Definir uma condição de desenvolvimento sustentável ou não consiste na operacionalização de um conceito e, para tanto, são propostos indicadores (SILVA; SOUZA-LIMA, 2010). Os indicadores são os reveladores das pautas de intervenção para garantir a saúde do ambiente e sua sustentabilidade.

No que se refere à temática ambiental, para Bellen (2006), devido às distintas definições de indicadores por diferentes autores, existe a necessidade, pela falta de consenso, de uma definição mais rigorosa e unificada para essa área. A grande maioria dos sistemas de indicadores existentes e utilizados foi desenvolvida por razões específicas: são ambientais, econômicos, de saúde e sociais e não podem ser considerados indicadores de sustentabilidade em si. Entretanto, eles muitas vezes possuem potencial representativo dentro do contexto do desenvolvimento sustentável. Portanto, assim como citado pelo autor mencionado, este estudo buscou por indicadores que além de se relacionarem com a saúde ambiental também pudessem ter potencial dentro de um contexto de sustentabilidade. 
Apesar da grande importância que a seleção de indicadores pode trazer como ferramenta de compreensão da realidade, deve-se observar que o presente estudo apresenta ainda algumas limitações.

O conjunto de indicadores selecionados está relacionado com a qualidade ambiental dos municípios, refletindo na sua saúde ambiental, ou seja, uma das dimensões que deve ser contemplada por um desenvolvimento urbano sustentável, entretanto, a conceituação do DS ainda é controversa e tanto o seu conceito como a composição de sua dimensionalidade refletem-se na eleição e elaboração de indicadores relativos à sua temática (PAULISTA et al., 2008).

A sustentabilidade é determinada por um conjunto de fatores (econômicos, sociais e ambientais) e quando se trata de indicadores ou índices de sustentabilidade, o debate está apenas se iniciando, pois não há, até o presente momento, uma fórmula ou receita consensual para avaliar o que é sustentável e o que é insustentável (SICHE et al., 2007).

Mesmo com as diferentes perspectivas acerca do tema, sistemas de indicadores para monitoramento e análise do processo de desenvolvimento sustentável por meio de abordagens utilizadas pela área ambiental vêm influenciando a criação e aplicação de modelos voltados para a saúde ambiental que procuram abordar a saúde e o ambiente como elementos fundamentais do processo de desenvolvimento (MALHEIROS et al., 2008).

Segundo Sobral e Freitas (2010), os problemas ambientais que se constituem em importantes elementos indutores da geração de doenças por meio da degradação dos ecossistemas e de seus serviços e também os efeitos das mudanças climáticas têm sido pouco considerados como determinantes ambientais da saúde em conjunto com os sociais. Segundo a Avaliação Ecossistêmica do Milênio (2005), os serviços ecossistêmicos são os benefícios que as pessoas obtêm dos ecossistemas. Estes incluem serviços de provisão tais como alimentos e água; regulação dos serviços tais como a regulação de inundações, seca, degradação da terra e doenças; serviços de apoio tais como formação do solo e ciclagem de nutrientes; além de serviços culturais, como de lazer, espiritual, religioso e outros benefícios não materiais. Portanto, o consenso é que áreas com extensa cobertura vegetal, em particular as de mata nativa localizadas nas regiões de mananciais ou de nascentes de cursos d'água de maior extensão, são prestadoras de serviços ambientais.

Nesse contexto, a expansão das áreas urbanas causa problemas como a perda de terrenos agrícolas e naturais, a fragmentação de florestas, a perda de zonas úmidas, poluição do ar, água e solo, além de problemas ambientais urbanos como a geração de resíduos, poluição sonora, congestionamentos, falta de água potável e demandas de energia que ameaçam seriamente a qualidade do ambiente, o bem-estar social e o desenvolvimento. 
Os desafios metropolitanos que se colocam nos dias atuais é que as cidades criem condições para assegurar uma qualidade de vida que possa ser considerada aceitável, não interferindo negativamente no meio ambiente do seu entorno e agindo preventivamente para evitar a continuidade do nível de degradação (JACOBI, 2006a).

Encontrar valores que englobem os aspectos ambientais da sustentabilidade exige a busca por indicadores não somente limitados a aspectos monetários, mas que incorporem as consequências da degradação ambiental, tais como os que estão associados às mudanças climáticas. Segundo Veiga (2010), indicadores para emissões de carbono que fossem bem calculados poderiam ser os indicadores das contribuições à insustentabilidade, assim como se surgissem medidas parecidas para o comprometimento dos recursos hídricos e para erosão da biodiversidade. Para o autor, talvez bastasse essa trinca de indicadores para mostrar a que distância se está do caminho da sustentabilidade.

Neste estudo, para identificar os municípios prestadores de serviços ambientais foi utilizado o indicador Percentual de Vegetação Nativa (IF, 2005). Entretanto, para esse tema, não foi utilizado qualquer indicador que identificasse a degradação dos ecossistemas. Com relação aos indicadores para emissões de carbono que contribuem para a insustentabilidade, como citados por Veiga (2010), foi incorporado o indicador Emissões de $\mathrm{CO}_{2}$ em milhões de toneladas, calculado pela Secretaria de Energia do Estado de São Paulo, mas não foi possível, como mencionado anteriormente, encontrar a trinca de indicadores proposta pelo autor.

A dificuldade de encontrar indicadores ambientais relacionados aos ecossistemas e aos recursos naturais decorre, segundo Freitas e colaboradores (2007a), devido à escassez de indicadores para essa área e à visão fragmentada da relação entre saúde e ambiente expressa pela pequena produção científica que considera a interface entre ecossistemas e a saúde humana. A integridade ecológica e a manutenção dos seus serviços são temas que precisam ser incorporados de forma mais contundente no âmbito da saúde pública.

Com relação aos indicadores de saúde, foram selecionados os mais tradicionais como os de saneamento (abastecimento de água, coleta de esgoto sanitário e coleta de lixo), de mortalidade e morbidade. Como puderam ser observados, muitos indicadores ambientais ainda são restritos à questão do saneamento. Outra dificuldade que vale ser mencionada foi de encontrar indicadores que pudessem corresponder ao tema da qualidade do ar, uma vez que a rede de monitoramento da Cetesb (Companhia Ambiental do Estado de São Paulo) não fornece dados de qualidade do ar para cada município, e sim valores gerais para a RMSP como um todo. Essa falta de homogeneidade de dados municipais pode refletir a falta de iniciativas ambientais nessa área, assim como dificultar a formulação de políticas públicas mais abrangentes no âmbito do território metropolitano. 
As últimas limitações a serem citadas são com relação ao período de tempo escolhido. Embora os dados selecionados representem o intervalo de tempo entre os anos de 2010 a 2015, a maioria dos indicadores selecionados correspondem somente ao ano de 2010, pois a maioria dos dados da análise é proveniente do Censo 2010 (IBGE, 2010).

Como não existem ainda indicadores construídos na perspectiva da sustentabilidade e também que contemplem a interface entre saúde e ambiente, os dados e indicadores disponíveis neste trabalho devem ser compreendidos assim como definido por Freitas e Giatti (2009), "algo similar a uma foto de uma região que é o resultado da colagem de diferentes partes de outras fotos da mesma região, tiradas em momentos distintos”. Os autores também citam que cada indicador é, inevitavelmente, uma redução da complexidade a um único e unidimensional valor.

De modo geral o presente trabalho esbarrou nas mesmas limitações apontadas por Freitas e colaboradores (2007b). Segundo os autores, a Saúde Pública enfrenta um enorme desafio na construção de sistemas de indicadores de sustentabilidade que integram as dimensões ambientais e do bem-estar humano, que está relacionado com os próprios limites dos dados disponíveis, principalmente quanto à produção de indicadores ambientais podendo ser citadas:

- as estatísticas ambientais são recentes no Brasil e ainda não estão disponíveis em nível municipal;

- ainda existem muitas incertezas sobre as mudanças na estrutura e na dinâmica dos ecossistemas e sua interação com o sistema socioambiental, que resulta em doenças, problemas de saúde e restrições ao bem-estar humano;

- quando os indicadores ambientais incorporam aspectos de saúde são geralmente limitados à morbidade e à mortalidade infantil e da expectativa de vida ao nascer;

- quando os indicadores de saúde incorporam aspectos ambientais são limitados a questões como qualidade da água para consumo humano e saneamento (acessar basicamente rede pública de esgoto e coleta de lixo).

Mesmo com as dificuldades mencionadas, no Brasil existem alguns estudos de análise da Matriz FPSEEA que conseguiram resultados positivos quando aplicados no campo da vigilância em saúde do trabalhar e na análise de riscos para atividades com potencial de impactos no ambiente e na saúde. Na perspectiva de riscos ambientais pode ser citado o trabalho de Araújo-Pinto e colaboradores (2012), que utilizaram a metodologia da matriz FPSEEA como um instrumento de análise de riscos ambientais e a saúde do ambiente associados com a intensa utilização de agrotóxicos nas diversas atividades agrícolas que são desenvolvidas no estado do Rio de Janeiro (RJ). Também podem ser citados os estudos de Mendes (2016), que analisou a matriz para levantar os impactos de hidrelétricas à saúde de pescadores e ribeirinhos e propor ações a partir dela no estado do Pará (PA), além do 
estudo de Rocha (2015), que utilizou a matriz para analisar os efeitos das queimadas na saúde da população de municípios da Amazônia Legal.

No campo da vigilância em saúde do trabalhador podem ser mencionados os trabalhos de Giardini et al. (2017), utilizando a matriz para avaliar riscos à saúde dos trabalhadores de postos de gasolina expostos ao benzeno em nível nacional e Quintino (2009) para vigilância em saúde dos trabalhadores da extração e beneficiamento de pedras ornamentais no estado de Minas Gerais (MG).

No Brasil é no âmbito da vigilância sanitária que a visão ambiental está mais consolidada e no qual o modelo FPSEEA é um bom instrumento auxiliar no levantamento e no fortalecimento de ações de intervenção e prevenção no processo saúde-doença. Como exemplo de aplicabilidade da matriz nesse sentido pode ser mencionado o estudo de Arantes e Pereira (2017), que selecionaram indicadores ambientais e socioeconômicos como subsídio para o fortalecimento das estratégias existentes no controle da dengue no município de Uberlândia (MG).

Utilizando a matriz para uma análise que adota um conceito ecossistêmico mais abrangente procurando ter o modelo FPSEEA como base na seleção de indicadores de sustentabilidade ambiental e saúde, um dos estudos precursores nesse campo é o trabalho desenvolvido por Giatti e colaboradores (2011). Esse estudo serviu de subsídio para a aplicação dessa mesma metodologia na Região Metropolitana de São Paulo (RMSP) no trabalho desenvolvido por Giatti e colaboradores (2013). Na RMSP destacam-se na aplicação do modelo FPSEEA os estudos de Silva (2015) e Maria (2015).

\section{Considerações Finais}

Medir e avaliar as relações entre a sociedade e a natureza por meio de indicadores constituise um problema complexo. Em comparação com os indicadores das áreas econômicas e sociais, que são clássicos e consolidados, o processo de escolha de indicadores ambientais aplicáveis ao ambiente urbano, que englobem os contextos de saúde e sustentabilidade, ainda se encontra distante de um consenso.

Mesmo com todas as limitações conceituais e operacionais, o modelo de indicadores FPSEEA deve ser utilizado como ferramenta auxiliar do modelo de determinação social da saúde. Entretanto, embora permita uma visão integrada dos indicadores, por si só não consegue contemplar toda a complexidade de inter-relações das dimensões que determinam e mediam o processo de produção social da saúde-doença. 
À medida que mais e melhores informações se tornem disponíveis nos sistemas públicos, espera-se que lacunas metodológicas possam ser melhoradas no futuro. Apesar de todas as limitações observadas, a iniciativa dos autores serviu de ponto de partida útil para a melhoria da metodologia inicial.

É importante também ressaltar que este estudo corrobora com o direito garantido pela Lei de Acesso à Informação, Lei n $12.527 / 2011$, que gera uma cidadania autônoma e fomenta a democracia participativa nas municipalidades.

Neste estudo, a matriz FPSEEA pôde ser aplicada como uma ferramenta auxiliar para a seleção dos indicadores de saúde ambiental, entretanto, para interpretar o dinamismo das dimensões da matriz é necessária a aplicação de análises estatísticas multivariadas do conjunto de dados e análises de séries temporais desses indicadores. Sendo assim, é uma área de investigação em início que requer a exploração de maiores estudos de aprimoramento dessas ferramentas.

A ferramenta metodológica desenvolvida é um passo inicial para reconhecer a macrometrópole, não só como uma expansão da metrópole, mas sim como um novo patamar de interação territorial funcional que requer uma gestão ambiental em nível regional.

\section{Agradecimentos}

À Coordenação de Aperfeiçoamento de Pessoal de Nível Superior (Fundação Capes) pelo financiamento da pesquisa.

\section{Referências}

ARANTES, K. M.; PEREIRA, B. B. Levantamento, análise e seleção de indicadores ambientais e socioeconômicos como subsídio para o fortalecimento das estratégias de controle da dengue no município de Uberlândia - MG. Journal of Health and Biological Sciences, v.5, n.1, p.86-94.

ARAÚJO-PINTO, M.; PERES, P.; MOREIRA, J. C. Utilização do modelo FPEEEA (OMS) para a análise dos riscos relacionados ao uso de agrotóxicos em atividades agrícolas do estado do Rio de Janeiro. Ciência \& Saúde Coletiva, v.17, n.6, p.1543-1555.

ASQUINO, M. S. A importância da Macrometrópole Paulista como escala de planejamento de infraestruturas de circulação e de transporte. Estudos Urbanos e Regionais, v.12, n.1, p.83-98. 2010. 
BELLEN, H. M. V. Indicadores de sustentabilidade - Uma análise comparativa. Rio de Janeiro: FGV, 2006.

CHAE, Y. Co-benefit analysis of an air quality management plan and greenhouse gas reduction strategies in the Seoul metropolitan area. Environmental Science \& Police,v. 13, n. 3, p. 205-216.

CORVALÁN, C.; BRIGGS D.; ZIELHUIS, G. Decision - Making in environmental health from evidence to action. Geneva: World Health Organization, 2000.

EMPLASA. Plano de ação da Macrometrópole. 2012. Disponível em: <http://www.emplasa.sp.gov.br/newsletter/maio/interno/acao_publica.asp>. Acesso em: maio 2015.

FREITAS, C. M. Problemas ambientais, saúde coletiva e ciências sociais. Ciência Saúde Coletiva, v.8, n.1, p.137-150, 2003.

FREITAS, C. M.; OLIVEIRA, S. G.; SCHÜTZ, G. S.; FREITAS, M. B.; CAMPONOVO, M. P. Enfoques ecossistêmicos e saúde: vertentes e aplicações na América Latina. Cadernos de Saúde Pública, v.23, n.2, fev. 2007a. Disponível em: <http://www.scielo.br/pdf/csp/v23n2/04.pdf>. Acesso em: out. 2014.

FREITAS, C. M.; SCHÜTZ, G. E.; OLIVEIRA, S. G. Indicadores de sustentabilidade ambiental e de bem-estar em perspectiva ecossistêmica na Região do Médio Paraíba, Rio de Janeiro, Brasil. Cadernos de Saúde Pública, v.23, supl. 4, p. 513-528, 2007b. Disponível em: <http://www.scielo.br/pdf/csp/v23s4/04.pdf>. Acesso em: out. 2014.

FREITAS, C. M.; GIATTI, L. L. Indicadores de sustentabilidade ambiental e de saúde na Amazônia Legal. Cadernos de Saúde Pública, v.25, n.6, p. 51-66, 2009. Disponível em: $<$ http//www.scielo.br/pdf/csp/v25n6/08.pdf>. Acesso em: nov. 2012.

GIARDINI, I.; POÇA, K. S.; SILVA, V. S. P.; MELLO, M. S. C.; FRIEDRICH, K. Vigilância sanitária em postos de revenda de combustíveis: aplicação de um modelo para integrar ações e promover a saúde do trabalhador. Revista Brasileira de Saúde Ocupacional, v.42, n.1, p.1-12, 2017. Acesso em: maio 2018.

GIATTI, L. G.; FREITAS, C. M.; DESMOULIÈRE, J. M.; MEDEIROS, M. S.; COSTA E SILVA, M. B.; NETO, A. L. S. Manaus: uma análise ecossistêmica através de indicadores de sustentabilidade ambiental e de saúde. In: Seminário Indicadores e Cenários Sustentabilidade Ambiental e de Saúde na Cidade de Manaus. Manaus: Fiocruz, 2011. p.1-53.

GIATTI, L. L; FREITAS, C. M.; NASCIMENTO, P. R.; LANDIN, R.; GAVIOLLI, J.; CUTOLO, S. A.; MARIA, N. C.; CARBONE, A. S.; TOLEDO, R. F. Estudo de distintos níveis holárquicos para uma região metropolitana por meio da aplicação de indicadores de sustentabilidade ambiental e de saúde. Revista Brasileira de Ciências Ambientais, n.30, p.79-88,2013.

INSTITUTO BRASILEIRO DE GEOGRAFIA E ESTATÍSTICA (IBGE). Indicadores de Desenvolvimento Sustentável. 2017. Disponível em:

<https://sidra.ibge.gov.br/pesquisa/ids/tabelas>. Acesso em: out. 2014. 
INSTITUTO FLORESTAL. Inventário florestal da vegetação natural do estado de São Paulo. 2005. Disponível em: <http://iflorestal.sp.gov.br/2005/03/01/inventario-florestal-da-vegetacaonatural-do-estado-de-sao-paulo/>. Acesso em: out. 2014

KICKBUSCH, I.; BUSS, P. M. Saúde na agenda pós-2015: perspectivas a meio do caminho. Cadernos de Saúde Pública, v.30, n.10, p.1-3, 2014.

JACOBI, P. Dilemas socioambientais na gestão metropolitana: do risco à busca da sustentabilidade urbana. Revista Política e Trabalho, n.25, p. 115-134, 2006a. Disponível em: <http://www.biblionline.ufpb.br/ojs2/index.php/politicaetrabalho/article/view/6742/4181>. Acesso em: out. 2013.

MALHEIROS, T. F.; PHLIPPI, A. J.; COUTINHO, S. M. V. Agenda 21 nacional e indicadores de desenvolvimento sustentável: contexto brasileiro. Saúde e Sociedade, v.17, n.1, p.7-20, jan-mar. 2008. Disponível em: <http://www.scielo.br/pdf/sausoc/v17n1/02.pdf.>. Acesso em: out. 2014.

MARIA, N. C. Uma tipologia em saúde ambiental para a Região Metropolitana de São Paulo: analisando dimensões de sustentabilidade. Dissertação de Mestrado (Mestrado em Ciências) Faculdade de Saúde Pública, Universidade de São Paulo, São Paulo, 2015.

MENDES, A. V. T. Impactos à saúde dos ribeirinhos em usina hidrelétrica do estado do Pará, Brasil. Monografia em Saúde Coletiva (Bacharelado) - Universidade de Brasília, Distrito Federal, 2016.

MILLENNIUM ECOSYSTEM ASSESSMENT. Ecosystems and human being - Synthesis. Washington DC: Island Press, 2005.

QUINTINO, N. D. Vigilância em saúde dos trabalhadores: potencialidades da matriz FPEEEA. Dissertação de Mestrado (Mestrado em Saúde Pública e Meio Ambiente) - Fundação Oswaldo Cruz, Rio de Janeiro, 2009.

PAULISTA, G.; VARVAKIS, G.; MONTIBELLER-FILHO, G. Espaço emocional e indicadores de sustentabilidade. Ambiente \& Sociedade, v.XI, n.1, p.185-200, jan-jun. 2008. Disponível em: <http://www.scielo.br/pdf/asoc/v11n1/12.pdf>. Acesso em: out. 2014.

PROGRAMA DAS NAÇÕES UNIDAS PARA O DESENVOLVIMENTO (PNUD). Os Objetivos de Desenvolvimento Sustentável. Disponível em: $<$ http://www.pnud.org.br/ODS.aspx $>$. Acesso em: ago.2015.

ROCHA, L. R. L. Desmatamento/queimadas e seus efeitos danosos à saúde da população nos municípios de Alta Floresta, Guarantã do Norte, Novo Mundo e Peixoto de Azevedo, na área e influência da BR-163, no estado do Mato Grosso. Tese de Doutorado (Doutorado em Ciências e Tecnologias em Saúde) - Universidade de Brasília, Distrito Federal, 2015.

SACHS, I. Desenvolvimento includente, sustentável, sustentado. Rio de Janeiro: Garamond, 2004.

SICHE, R.; AGOSTINHO, F.; ORTEGA, E.; ROMEIRO, A. Índices versus indicadores: precisões conceituais na discussão da sustentabilidade de países. Ambiente \& Sociedade, v.X, n.2, p.137-148, jul.-dez. 2007. Disponível em <http://www.scielo.br/pdf/asoc/v10n2/a09v10n2.pdf>. Acesso em: out. 2014. 
SILVA, A. A. Indicadores para avaliação de efeitos de intervenções de saneamento básico: a questão da sustentabilidade. Dissertação de Mestrado (Mestrado em Ciências) - Universidade de São Paulo, São Paulo, 2015.

SILVA, C. L.; SOUZA-LIMA, J. E. Políticas Públicas e indicadores para o desenvolvimento sustentável. São Paulo: Saraiva, 2010.

SILVA, R. T.; NUCCI, N. L. R.; COSTA, J. J. Recursos hídricos, saneamento e gestão metropolitana: os novos desafios. Brasil engenharia, p.103-110, 2012.

SOBRAL, A.; FREITAS, C. M. Modelo de Organização de Indicadores para Operacionalização dos Determinantes Socioambientais da Saúde. Saúde e Sociedade, v.19, n.1, p.35-37, 2010. Disponível em: 〈http://www.scielo.br/pdf/sausoc/v19n1/04.pdf>. Acesso em: out. 2014.

SOBRAL, A.; FREITAS, C. M.; BARCELlOS, C.; GURGEL H. C.; PEDROSO, M. M. Saúde Ambiental - Guia básico para a construção de indicadores. Brasília: Ministério da Saúde, 2011.

SECRETÁRIA DE RECURSOS HÍDRICOS DO ESTADO DE SÃO PAULO/DEPARTAMENTO DE ÁGUAS E ENEGIA ELÉTRICA DO ESTADO DE SÃO PAULO/COMPANHIA BRASILEIRA DE PROJETOS E EMPREENDIMENTOS. Macrométropole - Sumário Executivo. São Paulo. 2013.

STEINER, F. Urban human ecology. Urban Ecosystems,v.7, p.179-197, 2004.

TALAMINI, G. C.; GIATTI, L. L. Environmental health indicators for the Metropolitan Region of São Paulo, Brazil. Urban Environment, p.53-63, 2013.

VEIGA, J. E. Indicadores de sustentabilidade. Estudos Avançados, v. 24, n.68. p.39-52, 2010. Disponível em: <http://www.scielo.br/pdf/ea/v24n68/en_06.pdf>. Acesso em: out. 2014.

WU, J. Urban ecology and sustainability: The state-of-the-science and future directions. Landscape and Urban Planning,v.125, p.209-221, 2014.

Artigo recebido em 29/03/2018. Aceito para publicação em 08/06/2018. 